\title{
Pulsars at the Highest Energies: Questions for AGILE, Fermi (GLAST) and Atmospheric Cherenkov Telescopes
}

\author{
D. J. Thompson, on behalf of the Fermi Large Area Telescope Collaboration
}

NASA Goddard Space Flight Center, Greenbelt, MD 20771 USA

\begin{abstract}
Observational studies of gamma-ray pulsars languished in recent years, while theoretical studies made significant strides. Now, with new and improved gamma-ray telescopes coming online, opportunities present themselves for dramatic improvements in our understanding of these objects. The new facilities and better modeling of processes at work in highenergy pulsars should address a number of important open questions, some of which are summarized.
\end{abstract}

Keywords: gamma-ray telescopes, gamma-ray astrophysics, pulsars PACS: $95.55 . \mathrm{Ka}$, 95.85.Pw, 97.60.Gb

\section{INTRODUCTION}

Measurements of at least six high-energy gamma-ray pulsars by EGRET on the Compton Gamma Ray Observatory (for a summary, see Thompson, 2004), helped stimulate theoretical studies of how the rotational energy of these spinning neutron stars was being converted into accelerated particles and then into high-energy photons. These gamma rays offer a probe of the physics of the extreme conditions in a pulsar magnetosphere, with its high gravitational, electric, and magnetic fields. The de-orbiting of the Compton Observatory in 2000 left no satellite telescopes in the energy range above $10 \mathrm{MeV}$ where most of these high-energy pulsars had been found. Theoretical work continued, however, and now the convergence of new telescopes and improved modeling enables a resumption of progress in learning about gammaray pulsars.

\section{THE CHANGING OBSERVATIONAL ENVIRONMENT FOR HIGH-ENERGY PULSARS}

Two new gamma-ray telescopes are already in orbit, and steady progress on Atmospheric Cherenkov Telescopes (ACTs) allows these ground-based detectors to begin to detect pulsed gamma rays. The key developments, in chronological order:

- AGILE: First New Gamma-Ray Satellite Astro-rivelatore Gamma a Immagini LEggero (AGILE), a small satellite produced by an Italian collaboration, was launched from the Sriharikota launch facility in India on 23 April, 2007 (Tavani et al 2008). After more than a year of operation, it continues to function well in its near-equatorial orbit.
The primary gamma-ray instrument includes a pair production telescope and a relatively thin calorimeter. For photon energies above about $100 \mathrm{MeV}$, AGILE has an extremely large field of view, about 2.5 steradians. An important feature for pulsar studies is its accurate timing system, capable of $2 \mu \mathrm{s}$ absolute timing for individual gamma rays. AGILE is operated in a pointed mode, imaging large regions of the sky for up to several weeks continuously.

AGILE results on gamma-ray pulsars are beginning to appear (Pellizzoni et al 2008). The latest news about AGILE results can be found at http:// agile.rm.iasf.cnr.it/.

- MAGIC: Ground-based Gamma-ray Pulsar Detection

Until this year, the only hints of pulsed gamma-ray emission above $10 \mathrm{GeV}$ came from the handful of high-energy photons from EGRET (Thompson et al. 2005). The upper limits on the known gammaray pulsars from $\mathrm{TeV}$ telescopes were important, because they demonstrated that all the pulsar spectra had to roll over somewhere between the EGRET and ACT energy ranges.

The first step toward bridging the gap between ground and satellite telescopes came with the announcement that MAGIC detects pulsed emission above $25 \mathrm{GeV}$ from the Crab pulsar (Teshima, 2008). This result was enabled by the installation of a new low-energy triggering system. Some details of that result are given by Schweizer (2008).

MAGIC 2 and H.E.S.S. 2, with their larger detector areas, will push the ACT energy ranges down toward the range where additional gamma-ray pulsar detections, or at least better limits, may be possible. Prospects for pulsar work with ACTs appear promising. 
- Fermi Gamma-Ray Space Telescope: formerly Gamma-ray Large Area Space Telescope (GLAST) Another important step toward a renewed program of high-energy pulsar observations came on 11 June 2008, with the launch of the Gamma-ray Large Area Space Telescope (GLAST). GLAST was put into a circular orbit with $565 \mathrm{~km}$ altitude (96 min period)and a $25.6 \mathrm{deg}$ inclination. All spacecraft systems checked out and are functioning very well. The GPS information (position, time) is better than requirements by large factors. Both instruments, the Gamma-ray Burst Monitor (Meegan et al. 2008) and the Large Area Telescope (LAT, Atwood et al. 2008) are working nominally. The backgrounds appear to be close to expectation and very manageable. Following a 60 day checkout period, GLAST was renamed the Fermi Gamma-ray Space Telescope.

The Fermi Telescope is operated in a scanning mode, taking advantage of the very large fields of view of both instruments in order to scan the entire sky every three hours. With its high sensitivity, broad energy range ( $20 \mathrm{MeV}$ to more than 300 $\mathrm{GeV})$, good angular resolution, and excellent timing ( $1 \mu \mathrm{s}$ absolute), the LAT is well suited to finding new gamma-ray pulsars and measuring their properties with unprecedented accuracy.

\section{THE CHANGING THEORETICAL SITUATION FOR HIGH-ENERGY PULSARS}

High-energy pulsar theory has made substantial advances, building on previous results while waiting for new observations. Several particular developments have produced new tools and predictions to be tested.

\section{Re-emergence of the Slot Gap Model}

Much of the discussion about high-energy pulsars in recent years has concentrated on polar cap v. outer gap models, named for the part of the pulsar magnetosphere where particles are accelerated. Examples such as work by Hirotani (2008) and Harding, Usov and Muslimov (2005) demonstrate that both these models continue to advance. A third possibility, suggested by Arons and Scharlemann (1979) and Arons (1983), has now reemerged as an alternative.

The basic issue for any pulsar model is finding a region of charge depletion, because only in such a region can particles be accelerated. Figure 1 (Hirotani, 2008) shows a schematic of a pulsar magnetosphere, illustrating the possible locations for particle acceleration. The

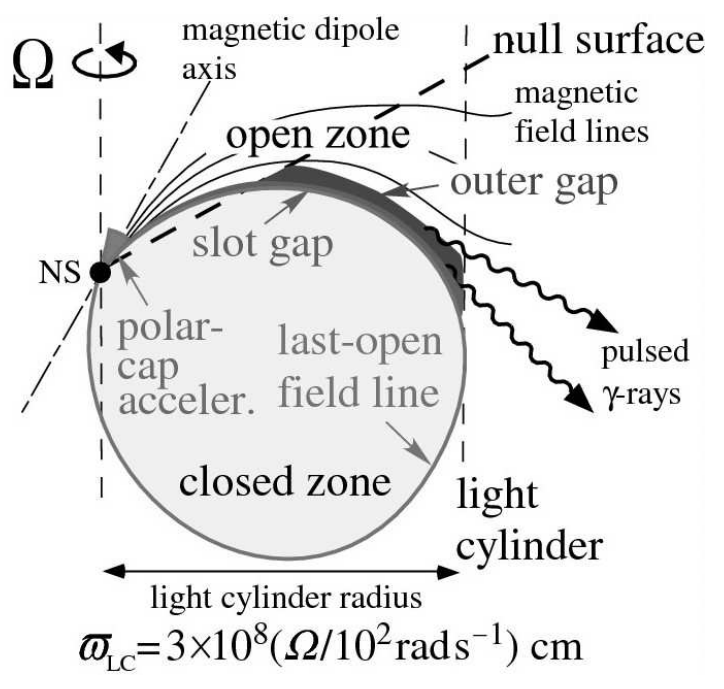

FIGURE 1. Particle accelerations regions in a pulsar magnetosphere (Hirotani, 2008).

new region, called the slot gap, is a narrow region right along the last open field line of the magnetic field. The slot gap can in principle extend from the surface of the star all the way to the speed of light cylinder, allowing either low-altitude or high-altitude particle acceleration and gamma-ray production. The slot gap opens a third alternative, with a different geometry than either of the other acceleration locations. A recent application of this model is described by Harding et al. (2008).

\section{Reconsideration of Caustics}

In classical optics, caustics are bright lines or spots produced by reflection or refraction of light by curved surfaces. Some early applications of the concept of caustics for pulsars were described by Morini (1983) and Romani and Yadigaroglu (1995), and more recently pulsar caustics were studied by Dyks and Rudak (2003) and Harding et al (2008). For pulsars, the curved surface is the last open field line. For the trailing edge of this rotating surface, emission tangent to this surface produces a bright peak, because time-of-flight (from the point where the radiation is emitted) and aberration (caused by the relativistic motion of the magnetic field in the outer magnetosphere) nearly cancel. The gamma rays emitted along much of this curved surface arrive at the observer at the same time, producing the pulse. Figure 2 shows the curved region where this effect is important.

In addition to producing a gamma-ray pulse from radiation occurring along an extended surface, caustics also allow radiation from above both poles of the neutron star to reach an observer. Two-pole models can then have 


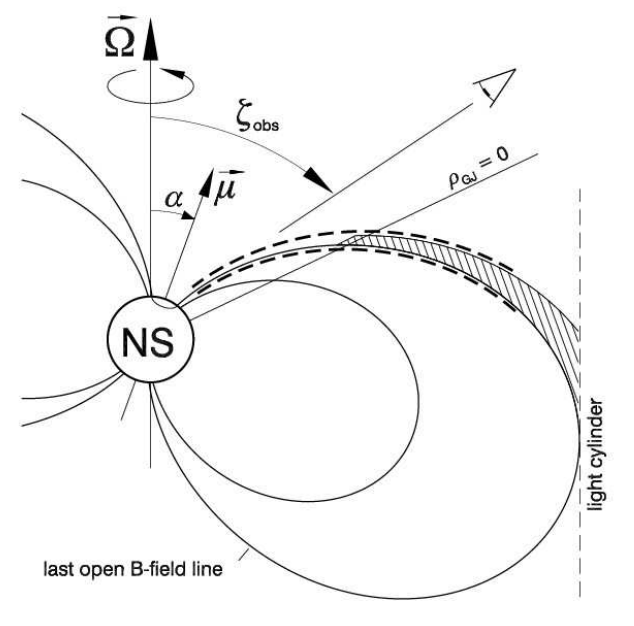

FIGURE 2. Another pulsar magnetosphere schematic, showing the curved region (between the dashed lines) that produces caustics (Dyks and Rudak, 2003).

peaks separated by less than $180^{\circ}$.

\section{Electrodynamics in Three Dimensions}

Although pulsars are clearly three-dimensional, many of the previous calculations of particle acceleration and interaction have been done in 1- or 2-dimensional approximation. In recent years, these calculations have been extended to the full 3-dimensional case. The basic equations relevant for gamma-ray pulsars are the Poisson equation, which describes the accelerating potential in the charge depletion region of the magnetosphere, and the Boltzmann equation, which calculates the particle transport and interaction processes. The essential series of steps is:

1. The strong electric potential in the charge depletion region accelerates particles to very high energies;

2. The energetic particles interact with the magnetic field through curvature radiation to produce highenergy gamma rays;

3. Some of the gamma rays interact with other photons or the strong magnetic field to produce electrons and positrons through pair production $\left(\gamma+\gamma=e^{+}+e^{-}\right.$ or $\left.\gamma+B=e^{+}+e^{-}\right)$;

4. Some of the electrons and positrons are then accelerated by the potential, and a shower of particles and photons develops.

These equations of particle acceleration/transport/interaction are now being solved, with boundary conditions, in full 3-dimensional geometry.
Some examples are Zhang et al. (2004), Dyks et al. (2004), Hirotani (2008), Harding et al. (2008). The increased realism of this calculation offers improved results on predictions of gamma-ray emission characteristics.

\section{Result: More Detailed Calculations of Radiation Patterns}

The combination of slot gap model calculations, caustics influence, and 3-dimensional electrodynamics has broadened the possibilities for explaining gamma-ray pulsar emission, while at the same time providing more detailed predictions that can be tested with the new generation of high-energy gamma-ray telescopes. Figure 3 shows an example of how these new calculations are applied. What is shown is the pattern of gamma radiation from the pulsar projected on the whole sky during a full rotation of the neutron star. A given observer sees the pulsar at one observing angle, the vertical scale. A horizontal line cutting across the radiation pattern produces the light curve expected for that particular observer. At different observing angles, the pulsar may appear with one pulse or multiple pulses from one or both poles and with varying relationships to the neutron star pole. Different models produce different radiation patterns that can be matched to the observations.

Two particular aspects of the predictive power of the new calculations are:

- Population studies

Modeling the Galactic population of neutron stars, taking into consideration the physical processes of gamma-ray production in different types of pulsars, and calculating the beaming expected for different models allows predictions of the gamma-ray pulsar populations to be found with the new telescopes. Gonthier et al (2007) have carried out such calculations. One prediction is that models dominated by outer magnetosphere emission are expected to result in a larger fraction of radio-quiet gamma-ray pulsars compared to those with emission closer to the polar caps.

- Phase-resolved spectra

The detailed radiation patterns produced by the current generation of pulsar models also give a more complete picture of phase-resolved spectra to be expected. Although the EGRET results showed evidence of different spectra for various pulsar phases (Fierro et al 1998), the uncertainties were large and only the brightest pulsars could be resolved. The more detailed predictions show that different patterns are expected for different models, providing a valuable discriminator among these models. 

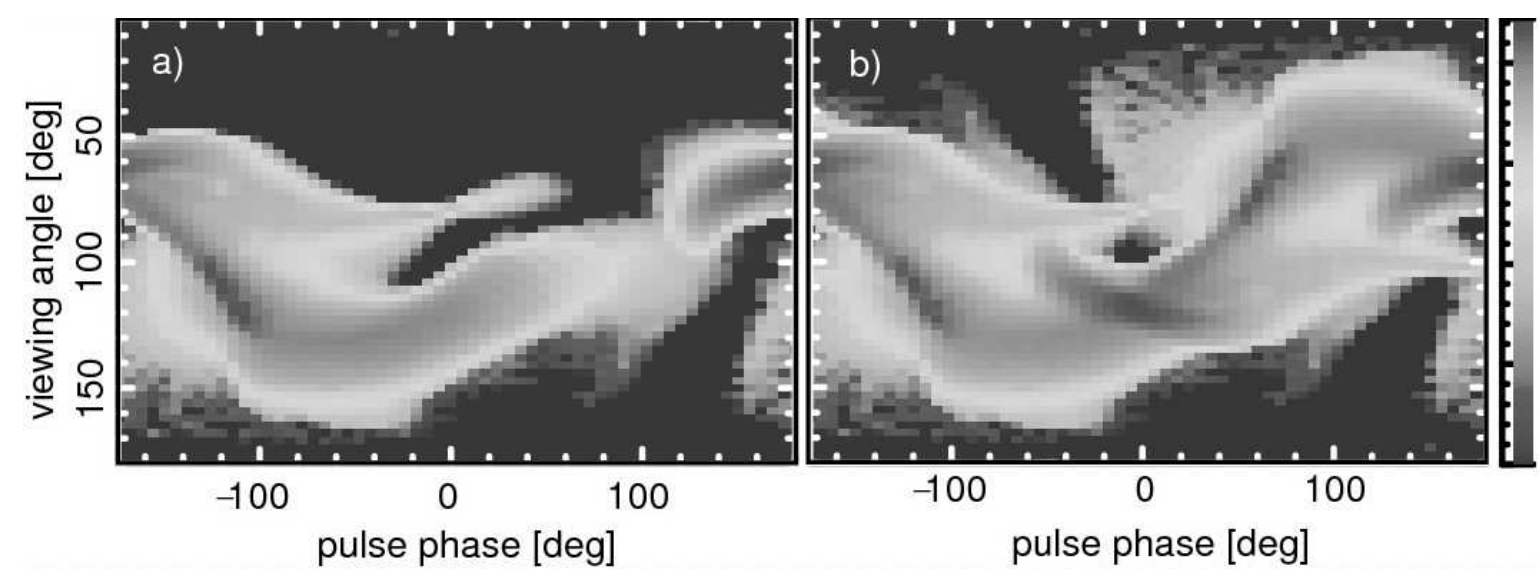

FIGURE 3. Pattern of gamma radiation projected onto the sky for the Crab pulsar in an outer gap model (Hirotani 2008). Left: pattern from one pole of the neutron star; right: emission from both poles.

\section{SOME OPEN QUESTIONS FOR THE NEAR FUTURE}

Gamma-ray pulsar studies have long viewed some fundamental questions as critical:

- Where and how are particles accelerated - polar cap, slot gap, outer gap?

- How do the particles interact? With what do they interact?

- Are the important processes the same for all neutron star systems?

- How does the complex environment (frame dragging, aberration, strong magnetic and electric fields, high currents) produce the observed radiation patterns?

- How can radio-quiet pulsar studies add to our knowledge of the Galaxy's neutron star population?

Answering such broad questions is not likely to come from one new set of measurements or one iteration of a pulsar model. A more practical approach is to look for some more tractable issues that can provide steps toward the larger ones. The following sections describe some specific questions that can plausibly be addressed within the next few years.

\section{Is the Crab Pulsar a Special Case?}

Among the known gamma-ray pulsars, the Crab is the youngest, has the highest spin-down luminosity, and converts the smallest fraction of that luminosity into gamma rays. It is also the only one whose light curve is similar across the electromagnetic spectrum. With the recent detection by MAGIC, it has become the first to be seen at energies above $20 \mathrm{GeV}$. Do all these unique features imply that the Crab is not a useful model for other pulsars but rather a special situation?

Some specific questions that can be asked about the Crab gamma-ray pulsar:

- Do models predict anything unusual about the Crab compared to other gamma-ray pulsars?

- Can we find more young pulsars with phase-aligned light curves?

- What are the details of the emission above $10 \mathrm{GeV}$ ? Is it different the highest-energy emission from other pulsars?

\section{Can Any Model Explain the Details of the Light Curves?}

Figure 4 illustrates the origin of this question. This summary by Alice Harding (from Grenier and Harding, 2006) shows the radiation patterns and derived light curves for the Vela gamma-ray pulsar in three models compared to the observed data. In the case of Vela, the observations are not statistics limited. The shape of the light curve is well defined. While all three of the models reproduce the general double-peak structure, none of them really matches the observations. Unexplained features include the shoulder following the first pulse and the broadening of the second pulse relative to the first. Since these features have been known since the COS-B era, the failure to reproduce them represents a challenge to all the models. 

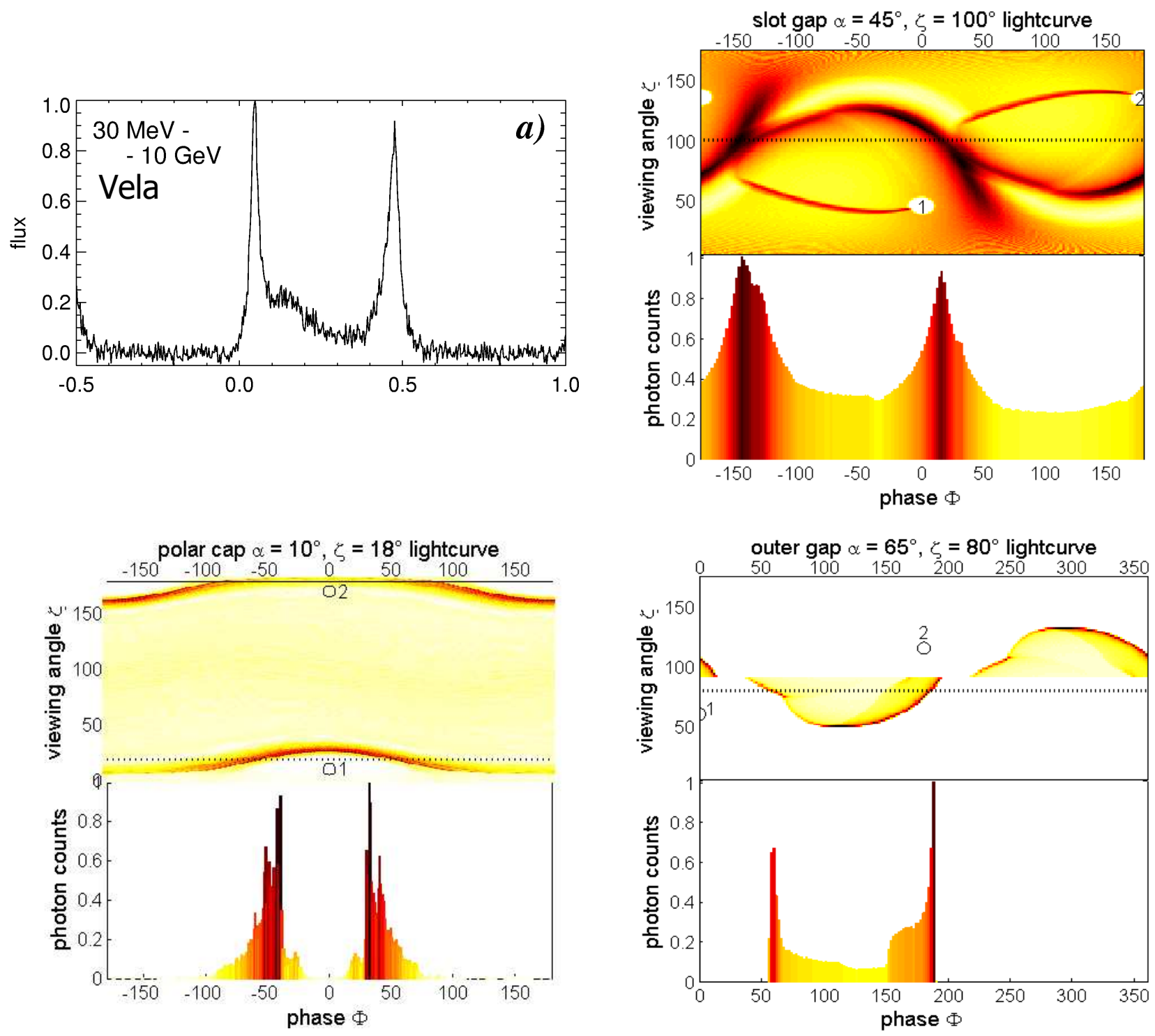

FIGURE 4. Light curves and radiation patterns for the Vela gamma-ray pulsar, from Grenier and Harding (2006). Upper left: observed EGRET light curve. Other figures show the results for three models. In each case, the dotted line in the upper panel shows the observer angle that produces the light curve shown in the lower panel. 


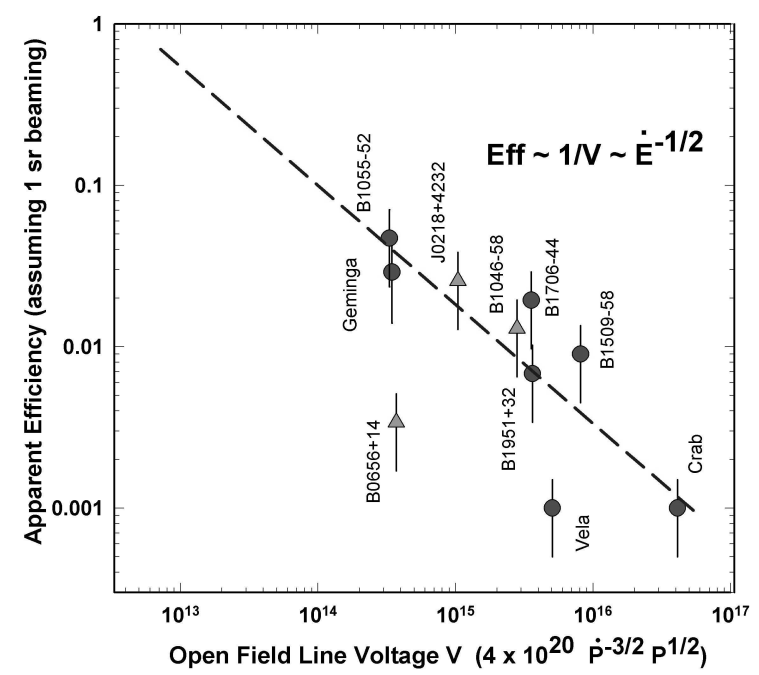

FIGURE 5. Gamma-ray pulsar efficiency as a function of open field line voltage.

\section{Do Observed Trends Extend to More Pulsars?}

On the basis of seven high-confidence and three lowerconfidence pulsars seen with the Compton Gamma Ray Observatory instruments, observers have constructed a variety of possible trends in various parameters. One example is shown in Figure 5. The apparent efficiency, gamma-ray luminosity/spin-down luminosity varies approximately inversely with the open field line voltage $\mathrm{V}$ (Arons, 1996). Although the data do not fit the pattern tightly and some outliers are apparent, the trend spans about two orders of magnitude. Will such trends persist when more gamma-ray pulsars are found?

Some specific questions are:

- What happens to pulsars with lower V as the efficiency approaches 1 ? Does the efficiency saturate, or do some pulsars just cease to radiate gamma rays? The trend is certainly not open-ended.

- How much effect does the beaming assumption have? The one steradian is a convenience, not a modeled value, and it is probably not valid for all pulsars. Large variations in the beaming would substantially alter this pattern.

\section{Can Fermi LAT Find Enough Radio-Quiet Pulsars to Test Population Models?}

Fermi LAT will have the capability to do blind searches for pulsars in cases where the gamma-ray source has sufficient signal to background. Simulations indicate that LAT is about an order of magnitude less sensitive to pulsations in a blind search than a search with a known timing solution. As discussed above, the number of new radio-quiet pulsars to be found is an important test of pulsar models. 3EG J1835+5918 is a prime candidate. The issue is whether the total number of such discoveries might be too small to be a statistically meaningful population.

\section{Will Observations and Models for Phase-Resolved Spectra Converge?}

Phase-resolved pulsar spectra offer valuable tests of pulsar models, but such tests require sufficiently good data to make a detailed comparison. Figure 6 shows an example of how a recent model predicts spectral variations with phase. With the large error bars on the data, particularly for weaker phase regions or extremes of the energy, the older data leave too much uncertainty for a valid comparison.

\section{Are Millisecond Pulsars Polar Cap Sources?}

The only EGRET indication for gamma-ray emission from a millisecond pulsar was a weak detection of PSR J0218+4232. Using a polar cap model, Harding, Usov, and Muslimov (2005) found an approach that can explain the observations, as shown in Figure 7. It uses cyclotron resonant absorption of radio emission to produce strong synchrotron gamma-ray emission. Other ms pulsars like J0437-4715 are predicted to have curvature radiation just below the EGRET limits, but easily visible to Fermi LAT. A possible problem is that this model seems to predict that EGRET should have seen some other ms pulsars. For comparison, the curvature radiation peak energy for an outer gap accelerator has been shown by Hirotani (2008) to be smaller than that for the polar cap, suggesting that very high-energy emission from a millisecond pulsar must come from the polar cap.

\section{CONCLUSIONS}

Within the next 2-3 years, new gamma-ray telescopes and improved theoretical modeling should answer many of the questions about gamma-ray pulsars left from the Compton Observatory era. In particular, observations should provide robust tests of phenomenological and theoretical models. 

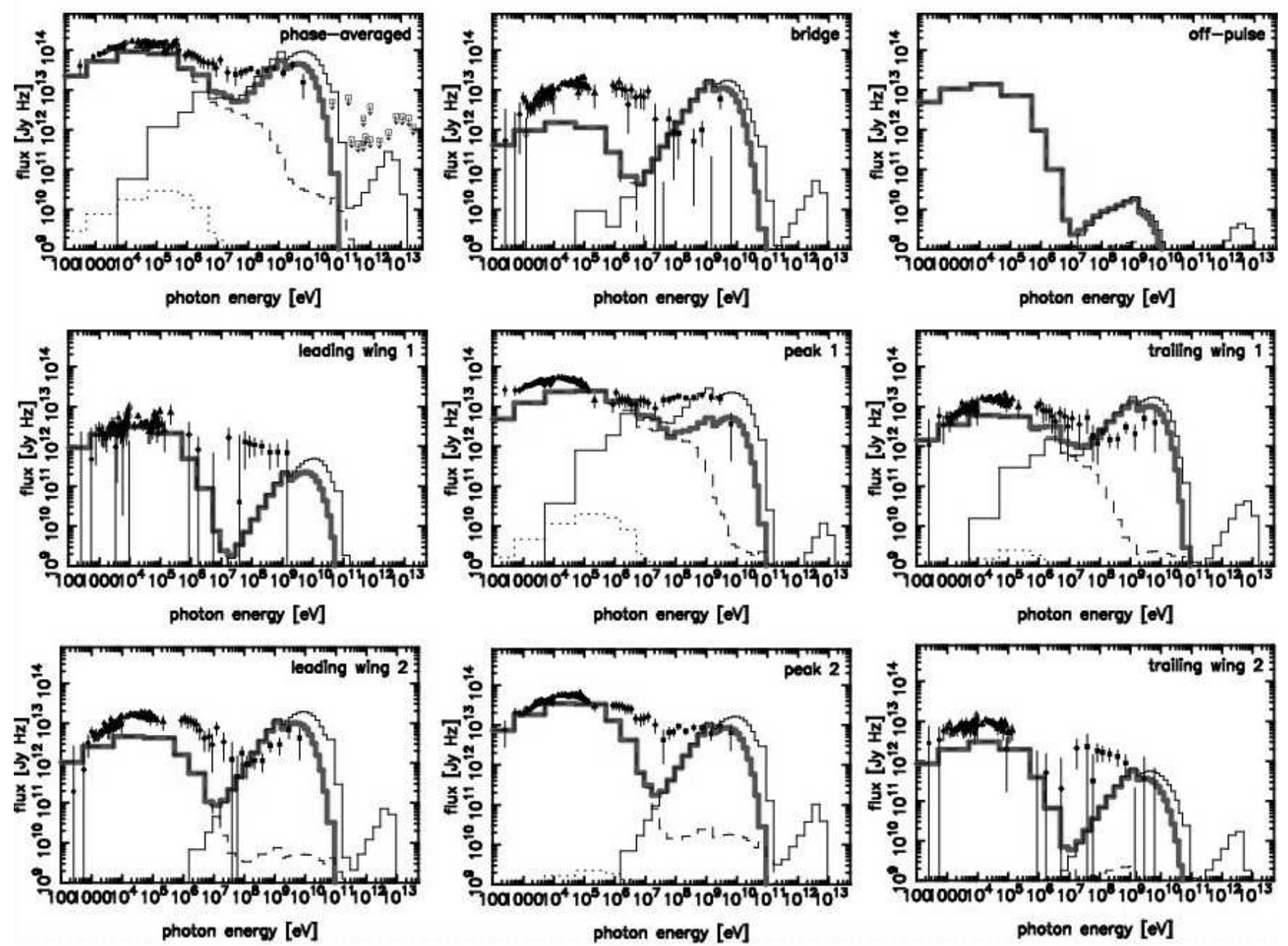

FIGURE 6. Outer gap model of phase-resolved spectra for high-energy emission from the Crab pulsar (Hirotani, 2008), compared with the EGRET data. The heavy line shows the calculated spectrum, including multiple emission components and absorption effects. 


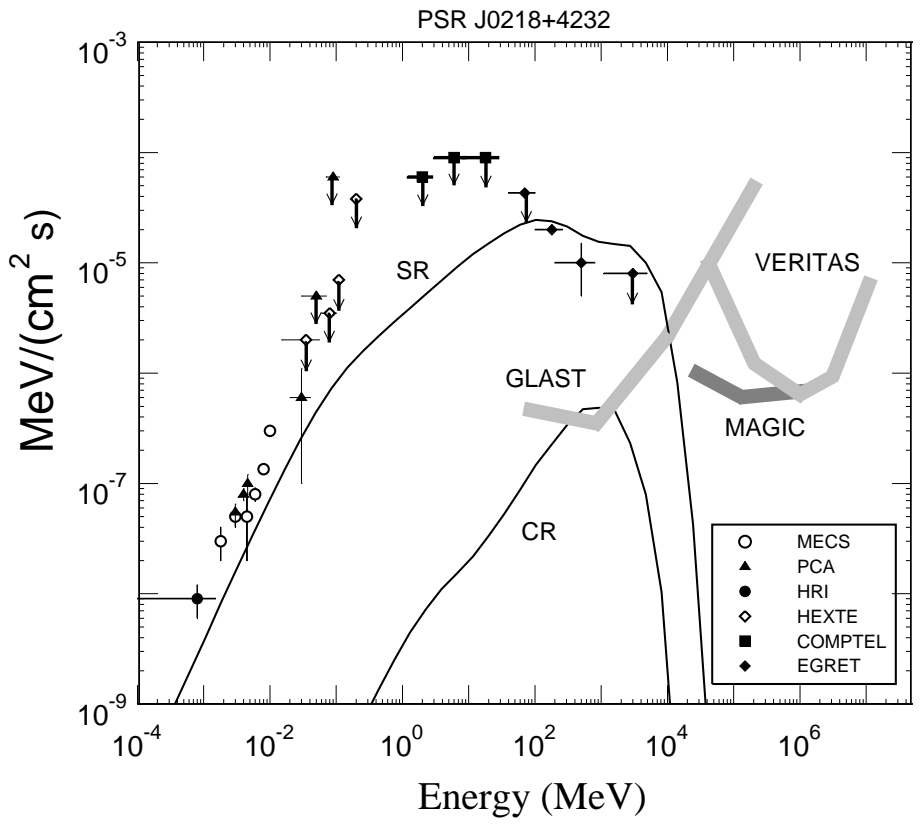

FIGURE 7. Polar cap model for gamma-ray emission from one millisecond pulsar (Harding, Usov, and Muslimov, 2005).

\section{ACKNOWLEDGMENTS}

I extend special thanks to Alice Harding for her help in preparing the material for this presentation.

\section{REFERENCES}

1. J. Arons , ApJ 266, 215-241 (1983).

2. J. Arons , AstrApS 120, 49-60 (1996).

3. J. Arons, E. T. Scharlemann, ApJ 231, 854-879 (1979).

4. W. B. Atwood et al., ApJ, submitted (2008).

5. J. Dyks, B. Rudak, ApJ 598, 1201-1206 (2003).

6. J. Dyks, A. K. Harding, B. Rudak, ApJ 606, 1125-1142 (2004).

7. J. Fierro, P. F. Michelson, P. L. Nolan, D. J. Thompson, ApJ 494, 734-746 (1998).

8. P. L. Gonthier, S. A. Story, B. D. Clow, A. K. Harding, ApSpSci 309, 245-251 (2007).

9. I. A. Grenier, A. K. Harding, "Pulsar Twinkling and Relativity," in Albert Einstein Century International Conference, AIP Conf. Proc. 861 2006, pp 630-637.

10. A. K. Harding, V. V. Usov, A. G. Muslimov, ApJ 622, 531-543 (2005).

11. A. K. Harding, J. V. Stern, J. Dyks, M. Frackowiak, ApJ 680, 1378-1393 (2008).

12. K. Hirotani, Open Astron. J., submitted (2008).

13. C. A. Meegan et al., ApJ, submitted (2008).

14. M. Morini, MNRAS 202, 495-510 (1983).

15. A. Pellizzoni, these proceedings.

16. R. Romani, I.-A. Yadigaroglu, ApJ 438, 314-321 (1995).

17. T. Schweizer, these proceedings.
18. S. A. Story, P. L. Gonthier, A. K. Harding, ApJ 671, 713-726 (2007).

19. M. Tavani et al., Nucl. Inst. Meth. A 588, 52-62 (2008).

20. M. Teshima., Astron. Tel. 1491, (2008).

21. D. J. Thompson, "Gamma Ray Pulsars: Multiwavelength Observations," in Cosmic Gamma-Ray Sources, ed. by K. S. Cheng and G. E. Romero, Kluwer, Dordrecht Boston London 2004, pp 149-168.

22. D. J. Thompson, D. L. Bertsch, R. H. O'Neal, Jr., ApJS 157, 324- 334, (2005).

23. L. Zhang, K. S. Cheng, Z. J. Jiang, P. Leung , ApJ 604, 317-327, (2004). 